\title{
Stratification du secteur anormal dans la sphère de Martinet de petit rayon
}

\author{
Bernard Bonnard ${ }^{1}$ and Emmanuel Trélat ${ }^{1}$ \\ Université de Bourgogne \\ Dpt de Mathématiques, UFR Sciences et Techniques \\ BP47870, 21078 Dijon Cedex, France
}

Résumé L'objectif de cet article est de fournir le cadre géométrique pour faire une analyse de la singularité de l'application exponentielle le long d'une direction anormale en géométrie sous-Riemannienne. Il utilise les calculs de [9], [12], et conduit dans le cas Martinet à une stratification de la singularité en secteurs Lagrangiens.

\section{Introduction}

Soit $M$ une variété analytique réelle et $F_{1}, F_{2}$ deux champs de vecteurs analytiques. Considérons le système :

$$
\frac{d q}{d t}=u_{1} F_{1}(q)+u_{2} F_{2}(q)
$$

où le contrôle $u=\left(u_{1}, u_{2}\right)$ est une application $L^{\infty}$ à valeurs dans un domaine $\mathcal{C}$. On considère le problème du temps minimal avec la contrainte $u_{1}^{2}+u_{2}^{2}=$ 1 (ou $\leqslant 1$ ). C'est équivalent au problème de minimiser la longueur d'une trajectoire de $(1)$ pour la métrique $: l(q)=\int_{0}^{T}\left(u_{1}^{2}+u_{2}^{2}\right)^{\frac{1}{2}} d t$ (problème sousRiemannien). On considère le système augmenté $: \dot{q}=u_{1} F_{1}(q)+u_{2} F_{2}(q)$, $\dot{q}_{0}=u_{1}^{2}+u_{2}^{2}$ et on appelle contrôle extrémal une singularité de l'application extrémité ( $T$ fixé) associée. Elles sont paramétrées par les équations :

$$
\dot{q}=\frac{\partial H_{\lambda}}{\partial p}, \dot{p}=-\frac{\partial H_{\lambda}}{\partial x}, \frac{\partial H_{\lambda}}{\partial u}=0
$$

où $H_{\lambda}(q, p, u)=<p, u_{1} F_{1}(q)+u_{2} F_{2}(q)>+\lambda\left(u_{1}^{2}+u_{2}^{2}\right),(p, \lambda) \neq(0,0)$ et $\lambda$ est une constante normalisée à 0 ou $-\frac{1}{2}$. On appelle extrémale une solution de ces équations et géodésique la projection d'une extrémale sur $M$. Elle est dite stricte si la singularité est de codimension un. On a deux types d'extrémales : les extrémales normales pour $\lambda=-\frac{1}{2}$, et les anormales pour $\lambda=0$. Ces dernières se projettent sur les trajectoires singulières du système 1 . On note $S(0, r)$ les points à distance $r>0$ de $q(0)=0$ pour la métrique sousRiemannienne, et $B(0, r)$ la boule sous-Riemannienne de rayon $r$.

La première étape de notre construction est de décomposer l'espace en une partie lisse et analytique et une partie non sous-analytique relativement au flot optimal. 


\section{La partie lisse et analytique}

Les trajectoires anormales sont solutions des équations :

$$
\frac{d q}{d t}=\frac{\partial H_{a}}{\partial p}, \quad \frac{d p}{d t}=-\frac{\partial H_{a}}{\partial q}
$$

où $H_{a}=u_{1} P_{1}+u_{2} P_{2}$, et : $u_{1}\left\{\left\{P_{1}, P_{2}\right\}, P_{1}\right\}+u_{2}\left\{\left\{P_{1}, P_{2}\right\}, P_{2}\right\}=0$, et sont contenues dans : $P_{1}=P_{2}=\left\{P_{1}, P_{2}\right\}=0$.

Notation. On identifie la condition initiale $q(0)=q_{0}$ à 0 . Soit $t \mapsto \gamma(t)$, $t \in]-T, T[$ une trajectoire de référence. On peut supposer qu'elle est associée au contrôle $u_{2}=0$. On fait alors l'hypothèse que les conditions suivantes sont vérifiées le long de $\gamma$ pour le couple $\left(F_{1}, F_{2}\right)$ :

$\left(H_{1}\right) K(t)=\operatorname{Vect}\left\{\operatorname{ad}^{k} F_{1} \cdot F_{2} \mid \gamma / k \in \mathbb{N}\right\}$ (premier cône de Pontriaguine) est de codimension un pour $t \in[0, T]$ et est engendré par les $n-1$ premiers éléments $\left\{F_{2}, \ldots, \text { ad }^{n-2} F_{1} . F_{2}\right\}_{\mid \gamma(t)}$.

$\left(H_{2}\right)\left\{P_{2},\left\{P_{1}, P_{2}\right\}\right\} \neq 0$ le long de $\gamma$.

$\left(H_{3}\right)$ Si $n \geqslant 3$, pour tout $t \in[0, T]$ on suppose que :

$$
F_{1 \mid \gamma} \notin\left\{\operatorname{ad}^{k} F_{1} \cdot F_{2 \mid \gamma} / k=0 \ldots n-3\right\}
$$

Sous ces hypothèses le vecteur adjoint $p_{\gamma}(0)$ est unique à un scalaire près. Identifions $M$ localement à un voisinage $U$ de 0 dans $\mathbb{R}^{n}$, et soit $V$ un voisinage de $p_{\gamma}(0)$ dans l'espace projectif $P\left(T_{0}^{*} U\right)$. On peut choisir $V$ assez petit de sorte que toutes les extrémales anormales issues de $\{0\} \times V$ vérifient les hypothèses $\left(H_{1}\right)-\left(H_{2}\right)-\left(H_{3}\right)$. On a :

Proposition 1. Sous les hypothèses $\left(H_{1}\right)-\left(H_{2}\right)-\left(H_{3}\right)$, il existe $r>0$ tel qu'une trajectoire anormale de longueur inférieure à $r$ soit stricte et globalement optimale.

On note $\Sigma_{r}$ le secteur de $U$ couvert par les extrémales anormales de longueur $\leqslant r$ issues de $\{0\} \times V$. La construction est visualisée sur la Fig.1.

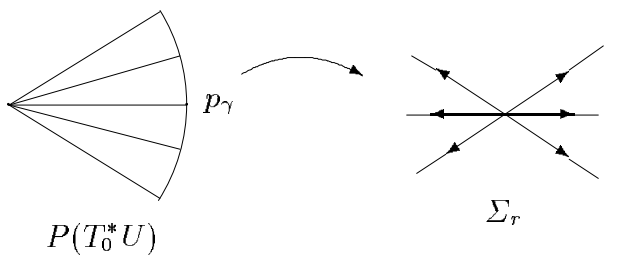

FIG.1.

Lemme 1 Pour $r$ assez petit, $\Sigma_{r}$ forme un secteur de la boule sous-Riemannienne homéomorphe à $C \cup(-C)$, où $C$ est un cône positif de dimension $n-2$. Sa trace sur la sphère est formée de deux surfaces analytiques de dimension $n-3$, réduite à deux points pour $n=3$. 


\section{Le lemme de recollement}

Proposition 2. (voir [12], [28]) Soit K(r) le cône de Pontriaguine évalué à l'extrémité $A$ de la trajectoire anormale de référence, de longueur $r, r$ assez petit. Alors $K(r)$ est un sous-espace vectoriel de codimension un et pour toute courbe lisse $\varepsilon \mapsto \alpha(\varepsilon), \alpha(0)=A, \varepsilon \geqslant 0$ telle que :

(i) $\alpha(\varepsilon) \subset S(0, r) \backslash \Sigma$ pour $\varepsilon \neq 0$

(ii) $\alpha(\varepsilon) \cap L(0) \neq \emptyset$ où $L$ est le lieu de coupure

l'espace tangent à la sphère évalué en $\alpha(\varepsilon)$ tend vers $K(r)$ lorsque $\varepsilon \rightarrow 0$.

Corollaire 2 Avec les notations de la proposition précédente, $\alpha(\varepsilon)$ n'est pas l'image d'une partie compacte du cylindre $P_{1}^{2}(0)+P_{2}^{2}(0)=\frac{1}{2}$. L'application exponentielle n'est donc pas propre le long de l'anormale.

Une analyse plus fine utilise les estimations calculées dans le cas Martinet qui est le suivant. On considère la structure sous-Riemannienne $(U, D, g)$ où $U$ est un ouvert de $\mathbb{R}^{3}$ contenant $0, D=\operatorname{Ker} \omega, \omega=d z-\frac{y^{2}}{2} d x, q=(x, y, z)$ et $g=a(q) d x^{2}+c(q) d y^{2}, a, c$ étant des germes de fonctions analytiques en 0 tels que $a(0)=c(0)=1$. On note $: G_{1}=\frac{\partial}{\partial x}+\frac{y^{2}}{2} \frac{\partial}{\partial z}, G_{2}=\frac{\partial}{\partial y}$. Le repère orthonormé est : $F_{1}=\frac{G_{1}}{\sqrt{a}}, F_{2}=\frac{G_{2}}{\sqrt{c}}, \quad F_{3}=\frac{\partial}{\partial z}$, où $F_{1}, F_{2}$ engendrent $D$.

Sans nuire à la généralité de notre analyse, on peut supposer le problème isopérimétrique, c'est-à-dire que la métrique $g$ ne dépend pas de $z$. Les géodésiques anormales se projettent dans le plan $y=0$ et sont des droites $z=z_{0}$. La ligne anormale $L$ issue de 0 et paramétrée par la longueur est $L=( \pm t, 0,0)$. Pour $i=1,2,3$ on pose : $P_{i}=\left\langle p, F_{i}>\right.$. Dans les coordonnées $(q, P)$ les extrémales normales sont solutions des équations :

$$
\begin{aligned}
\dot{x} & =\frac{P_{1}}{\sqrt{a}}, \dot{y}=\frac{P_{2}}{\sqrt{c}}, \dot{z}=\frac{y_{2}}{2} \frac{P_{1}}{\sqrt{a}} \\
\dot{P}_{1} & =\frac{P_{2}}{\sqrt{a} \sqrt{c}}\left(y P_{3}-\frac{a_{y}}{2 \sqrt{a}} P_{1}+\frac{c_{x}}{2 \sqrt{c}} P_{2}\right), \dot{P}_{2}=-\dot{P}_{1}, \dot{P}_{3}=0
\end{aligned}
$$

En paramétrant par la longueur $H_{n}=\frac{1}{2}\left(P_{1}^{2}+P_{2}^{2}\right)=\frac{1}{2}$ et en introduisant les coordonnées cylindriques : $P_{1}=\cos \theta, P_{2}=\sin \theta, P_{3}=\lambda$, on obtient les équations suivantes :

$$
\dot{x}=\frac{P_{1}}{\sqrt{a}}, \quad \dot{y}=\frac{P_{2}}{\sqrt{c}}, \quad \dot{z}=\frac{y^{2}}{2} \frac{P_{1}}{\sqrt{a}}, \quad \dot{\theta}=-\frac{1}{\sqrt{a} \sqrt{c}}\left(\lambda y-\frac{a_{y}}{2 \sqrt{a}} P_{1}+\frac{c_{x}}{2 \sqrt{c}} P_{2}\right)
$$

Les variables $x, y, z$ sont graduées en fonction de la règle de [7] avec les poids suivants : le poids de $x, y$ est un et le poids de $z$ est trois. La forme normale graduée est :

- ordre $-1: g=d x^{2}+d y^{2}$ (cas plat) 
- ordre 0: $g=a d x^{2}+c d y^{2}$ avec $a=(1+\alpha y)^{2}$ et $c=(1+\beta x+\gamma y)^{2}$

et à l'ordre 0 les équations précédentes s'écrivent :

$$
\dot{x}=\frac{\cos \theta}{\sqrt{a}}, \dot{y}=\frac{\sin \theta}{\sqrt{c}}, \dot{z}=\frac{y^{2}}{2} \frac{\cos \theta}{\sqrt{a}}, \dot{\theta}=\frac{1}{\sqrt{a} \sqrt{c}}(\lambda y-\alpha \cos \theta+\beta \sin \theta)
$$

En introduisant le paramétrage $\sqrt{a} \sqrt{c} \frac{d}{d t}=\frac{d}{d \tau}$, les équations se projettent dans le plan $(y, \theta)$ en : $y^{\prime}=\sqrt{a} \sin \theta, \theta^{\prime}=-(\lambda y-\alpha \cos \theta+\beta \sin \theta)$, où ' désigne la dérivée par rapport à $\tau$, et le système équivaut à :

$$
\theta^{\prime \prime}+\lambda \sin \theta+\alpha^{2} \sin \theta \cos \theta-\alpha \beta \sin ^{2} \theta+\beta \theta^{\prime} \cos \theta=0
$$

La condition initiale $q(0)=0$ induit la contrainte $y=0$ qui se traduit par la condition : $\theta^{\prime}=\alpha \cos \theta-\beta \sin \theta$.

Pour ces calculs, le cas général est interprété comme une déformation $d u$ cas plat, bien que dans le cas plat la direction anormale ne soit pas stricte. En effet dans les équations précédentes, en paramétrant les solutions par $\tau \sqrt{\lambda}$, on transforme les paramètres $\alpha, \beta, \gamma$ de la métrique en petits paramètres. Cela revient à étudier la sphère sous-Riemannienne dans un $C^{0}$-voisinage de la trajectoire de référence.

Dans la section suivante on relève le problème sur le groupe d'Engel pour avoir une représentation uniforme.

\section{Le cas Martinet relevé sur le groupe d'Engel}

Si $q=(x, y, z, w)$, on considère le système de $\mathbb{R}^{4}$ :

$$
F_{1}=\frac{\partial}{\partial x}+y \frac{\partial}{\partial z}+\frac{y^{2}}{2} \frac{\partial}{\partial w}, \quad F_{2}=\frac{\partial}{\partial y}
$$

On a les relations : $F_{3}=\left[F_{1}, F_{2}\right]=\frac{\partial}{\partial z}+y \frac{\partial}{\partial w}, F_{4}=\left[\left[F_{1}, F_{2}\right], F_{1}\right]=\frac{\partial}{\partial w}$ et $\left[\left[F_{1}, F_{2}\right], F_{1}\right]=0$. Par ailleurs tous les crochets de Lie de longueur $\geqslant 4$ sont nuls. On pose :

$$
L_{1}=\left(\begin{array}{llll}
0 & 0 & 0 & 0 \\
0 & 0 & 1 & 0 \\
0 & 0 & 0 & 1 \\
0 & 0 & 0 & 0
\end{array}\right), \quad L_{2}=\left(\begin{array}{llll}
0 & 1 & 0 & 0 \\
0 & 0 & 0 & 0 \\
0 & 0 & 0 & 0 \\
0 & 0 & 0 & 0
\end{array}\right)
$$

et on définit la représentation : $\rho\left(F_{1}\right)=L_{1}, \rho\left(F_{2}\right)=L_{2}$ qui permet d'identifier le système sur $\mathbb{R}^{4}$ au système invariant à gauche $\dot{R}=\left(u_{1} L_{1}+u_{2} L_{2}\right) R$ sur le groupe d'Engel $G_{e}$, représenté ici par les matrices nilpotentes :

$$
\left(\begin{array}{cccc}
1 & q_{2} & q_{3} & q_{4} \\
0 & 1 & q_{1} & \frac{q_{1}^{2}}{2} \\
0 & 0 & 1 & q_{1} \\
0 & 0 & 0 & 1
\end{array}\right)
$$


Le poids de $x, y$ est un, le poids de $z$ deux, et le poids de $w$ est trois. Pour toute métrique sous-Riemannienne sur $G_{e}$, l'approximation d'ordre - 1 est la métrique plate $g=d x^{2}+d y^{2}$. Toute métrique sous-Riemannienne de Martinet s'écrit $g=a d x^{2}+c d y^{2}$ et se relève sur le groupe $G_{e}$.

Paramétrage des géodésiques dans le cas plat. Les extrémales anormales non triviales sont solutions de :

$$
P_{1}=P_{2}=\left\{P_{1}, P_{2}\right\}=0, u_{1}\left\{\left\{P_{1}, P_{2}\right\}, P_{1}\right\}+u_{2}\left\{\left\{P_{1}, P_{2}\right\}, P_{2}\right\}=0
$$

En posant $p=\left(p_{x}, p_{y}, p_{z}, p_{w}\right)$ on obtient :

$$
p_{x}+p_{z} y+p_{w} \frac{y^{2}}{2}=p_{y}=p_{z}+y p_{w}=p_{w} u_{2}=0
$$

Cela implique $p_{w} \neq 0$ et donc $u_{2}=0$. Le flot anormal est donné par :

$$
\dot{x}=u_{1}, \quad \dot{y}=0, \quad \dot{z}=u_{1} y, \quad \dot{w}=u_{1} \frac{y^{2}}{2}
$$

où $\left|u_{1}\right|=1$ si on paramètre par la longueur.

Pour calculer les extrémales normales, on pose $P_{i}=<p, F_{i}(q)>, i=$ $1,2,3,4$ et $H_{n}=\frac{1}{2}\left(P_{1}^{2}+P_{2}^{2}\right)$; on obtient :

$$
\dot{P}_{1}=P_{2} P_{3}, \quad \dot{P}_{2}=-P_{1} P_{3}, \quad \dot{P}_{3}=P_{2} P_{4}, \quad \dot{P}_{4}=0
$$

En paramétrant par la longueur $H_{n}=\frac{1}{2}$, on peut poser : $P_{1}=\cos \theta, P_{2}=$ $\sin \theta$, et pour $\theta \neq k \pi$ il vient $: \dot{\theta}=-P_{3}, \ddot{\theta}=-P_{2} P_{4}$. En notant $P_{4}=\lambda$, cela équivaut à l'équation du pendule :

$$
\ddot{\theta}+\lambda \sin \theta=0
$$

On désigne par $L$ la ligne anormale issue de $0: t \mapsto( \pm t, 0,0,0)$. Elle n'est pas stricte et se projette en $\theta=k \pi$.

Pour obtenir une représentation uniforme des géodésiques normales, on utilise la fonction elliptique de Weierstrass $\mathcal{P}$. En effet le système admet trois intégrales premières : $P_{1}^{2}+P_{2}^{2}=1$, et deux Casimir : $-2 P_{1} P_{4}+P_{3}^{2}=C$ et $P_{4}=\lambda$. En utilisant $\dot{P}_{1}=P_{2} P_{3}$ on obtient : $\ddot{P}_{1}=-C P_{1}-3 \lambda P_{1}^{2}+\lambda$, qui équivaut pour $\dot{P}_{1} \neq 0$ et $\lambda \neq 0$ à l'équation : ${\dot{P_{1}}}^{2}=-\lambda\left(P_{1}^{3}+\frac{C}{2 \lambda} P_{1}^{2}-P_{1}-\frac{C}{2 \lambda}\right)$. Soit $\mathcal{P}(u)$ la fonction elliptique de Weierstrass (cf [20]) solution de :

$$
\mathcal{P}^{\prime}(u)=-2 \sqrt{\left(\mathcal{P}(u)-e_{1}\right)\left(\mathcal{P}(u)-e_{2}\right)\left(\mathcal{P}(u)-e_{3}\right)}
$$

où les complexes $e_{i}$ vérifient $e_{1}+e_{2}+e_{3}=0$. En posant $g_{2}=-4\left(e_{2} e_{3}+\right.$ $\left.e_{3} e_{1}+e_{1} e_{2}\right)$ et $g_{3}=4 e_{1} e_{2} e_{3}$ on peut l'écrire : $\mathcal{P}^{\prime}(u)=4 \mathcal{P}^{3}(u)-g_{2} \mathcal{P}(u)-g_{3}$, la fonction $\mathcal{P}(u)$ étant développable en 0 selon :

$$
\mathcal{P}(u)=\frac{1}{u^{2}}+\frac{1}{20} q_{2} u^{2}+\frac{1}{28} q_{3} u^{4}+\cdots
$$


La solution $P_{1}$ peut donc s'exprimer sous la forme $a \mathcal{P}(u)+b$. On peut ensuite calculer $P_{2}$ et $P_{3}$ en utilisant les intégrales premières, et $x, y, z, w$ se calculent par quadratures. On peut retrouver les solutions oscillantes et en rotation du pendule en utilisant les fonctions elliptiques de Jacobi données par les formules :

$$
\operatorname{cn} u=\left(\frac{\mathcal{P}(u)-e_{1}}{\mathcal{P}(u)-e_{2}}\right)^{\frac{1}{2}}, \operatorname{dn} u=\left(\frac{\mathcal{P}(u)-e_{2}}{\mathcal{P}(u)-e_{3}}\right)^{\frac{1}{2}}
$$

\section{Les cas Heisenberg et Martinet plat déduits du cas Engel. Eclatement en droites}

On observe que les deux champs $\frac{\partial}{\partial z}$ et $\frac{\partial}{\partial w}$ commutent avec $F_{1}$ et $F_{2}$. Le cas Engel contient le cas de contact plat et le cas Martinet plat restitués par les opérations suivantes :

- En posant $p_{z}=0$, on obtient les géodésiques du cas Heisenberg.

- En posant $p_{w}=0$, on obtient les géodésiques du cas Martinet plat. L'interprétation est la suivante.

Lemme 3 On obtient le cas Martinet plat (resp. Heisenberg) en minimisant la distance sous-Riemannienne par rapport à la droite $(O z)$ (resp. $(O w))$.

En effet la condition $p_{z}=0$ (resp. $p_{w}=0$ ) correspond alors à la condition de transversalité. Il est intéressant de noter que comme la distance sousRiemannienne par rapport à une droite est plus régulière que par rapport à un point, la distance sous-Riemannienne d'Engel hérite donc au moins de toutes les singularités du cas Heisenberg et du cas Martinet plat.

Une autre façon de déduire le cas Martinet plat est d'utiliser le résultat général de [7] :

Lemme 4 Le cas Martinet plat est isométrique à $\left(G_{e / H}, d x^{2}+d y^{2}\right)$ où $H$ est le sous-groupe de $G_{e}:\left\{\exp t\left[F_{1}, F_{2}\right] / t \in \mathbb{R}\right\}$.

\section{Stratification dans le cas Martinet}

On rappelle le paramétrage explicite des géodésiques, cf [4].

Proposition 3. Les géodésiques normales issues de 0 paramétrées par la longueur sont données par:

- $\lambda \neq 0, u=K+t \sqrt{\lambda}$

$$
\begin{aligned}
& x(t)=-t+\frac{2}{\sqrt{\lambda}}(E(u)-E(K)), y(t)=-\frac{2 k}{\sqrt{\lambda}} \text { cn } u \\
& z(t)=\frac{2}{3 \lambda^{\frac{3}{2}}}\left(\left(2 k^{2}-1\right)(E(u)-E(K))+{k^{\prime}}^{2} t \sqrt{\lambda}+2 k^{2} \text { sn u cn } u \text { dn } u\right)
\end{aligned}
$$


où sn $u, c n u, d n u$ et $E(u)$ sont les fonctions elliptiques de Jacobi, et $K, E(K)$ sont les intégrales complètes.

- $\lambda=0$

$$
\left.\left.x(t)=t \sin \phi, y(t)=t \cos \phi, \quad z(t)=\frac{t^{3}}{6} \sin \phi \cos ^{2} \phi \quad \text { où } \phi \in\right]-\frac{\pi}{2}, \frac{\pi}{2}\right]
$$

et les courbes déduites des précédentes en utilisant les symétries $S_{1}:(x, y, z) \mapsto$ $(x,-y, z)$ et $S_{2}:(x, y, z) \mapsto(-x, y,-z)$.

Chaque extrémale normale est minimisante jusqu'à son premier retour en $y=0$, c'est-à-dire pour $t \sqrt{\lambda} \leqslant 2 K$. Le premier temps conjugué vérifie $2 K<t_{1 c} \sqrt{\lambda}<3 K$, et plus précisément une simulation numérique montre que $\frac{t_{1 \mathrm{c}} \sqrt{\lambda}}{3 K} \simeq 0.97$.

Conséquences. Le module $k$ des fonctions de Jacobi est donné par : $k^{2}=$ $\frac{1-P_{1}(0)}{2}$. Lorsque $k \rightarrow 0, K(k) \rightarrow \frac{\pi}{2}$, et lorsque $k^{2}=1-k^{2} \rightarrow 0, K(k) \sim$ $\ln \frac{1}{k^{\prime}}$. La trace de la sphère avec le plan de Martinet et pour $z \geqslant 0$ est représentée Fig.2 ; elle forme l'adhérence du lieu de partage. Le corollaire 2 ne s'applique pas car la géodésique anormale n'est pas stricte, néanmoins l'application exponentielle n'est pas propre. La sphère est sous-analytique en $B$ mais pas en $A$. Dans ce cas dégénéré, l'application exponentielle applique tout le bord du cylindre sur la direction anormale. Lorsque $\lambda \rightarrow \infty$, les points de coupure et les points conjugués s'accumulent le long de la direction anormale.

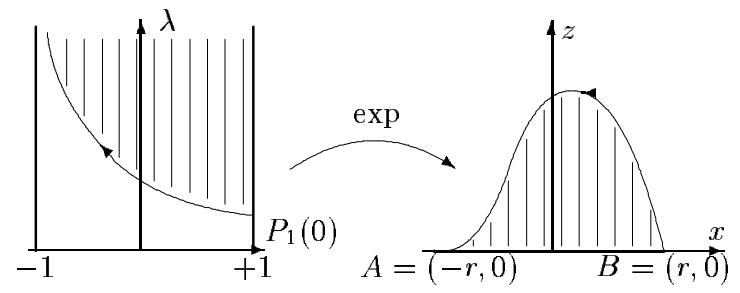

FIG.2.

Le cas générique. Pour étudier la situation générale on utilise la forme graduée d'ordre 0 de la section 2. Elle dépend de trois paramètres $\alpha, \beta, \gamma$ dont le rôle géométrique est le suivant :

Lemme 5 Pour la forme normale graduée d'ordre $0: g=(1+\alpha y)^{2} d x^{2}+$ $(1+\beta x+\gamma y)^{2} d y^{2}$, on $a$ :

1. La trajectoire anormale est stricte si et seulement si $\alpha \neq 0$.

2. Le pendule (3) est conservatif si et seulement si $\beta=0$. Dans ce cas, le flot géodésique est intégrable par quadratures en utilisant les fonctions elliptiques de Jacobi. 
Par ailleurs les calculs de [9] conduisent à la conjecture suivante :

Conjecture. Les premiers points conjugués localisés au voisinage de la direction anormale sont avant le troisième retour d'une géodésique sur le plan de Martinet.

C'est en fait un résultat qui permet de compactifier notre analyse car on ne considère que des trajectoires avec un nombre uniformément borné d'oscillations (comparer avec [1]).

Trace de la sphère avec le plan $y=0$, au voisinage de l'anormale, si $\alpha \neq 0$ (cas strict) Elle est représentée Fig.3.

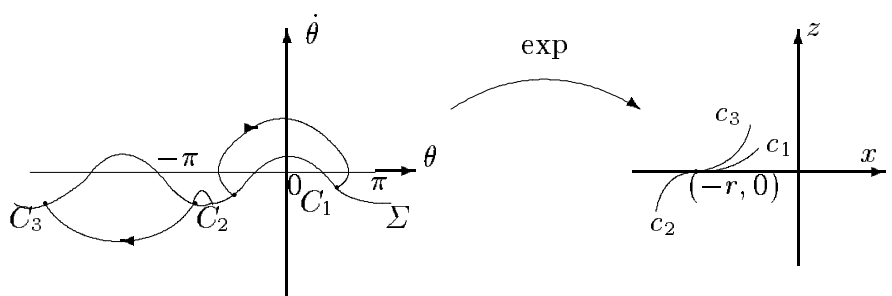

FIG.3.

La section $\Sigma$ correspond à la projection de $y=0$ dans le plan de phase du pendule. Les trois courbes $c_{1}, c_{2}, c_{3}$ se ramifient sur la direction anormale et sont construites ainsi : la courbe $c_{2}$ est associée à des petits déplacements localisés au voisinage du col; les courbes $c_{1}, c_{3}$ correspondent à de grands déplacements et il y a deux courbes car il faut considérer les trajectoires oscillantes du pendule (courbe $c_{1}$ ) et les trajectoires en rotation (courbe $c_{3}$ ). Dans le cas plat, $\Sigma$ est la droite $\dot{\theta}=0$, et seule la branche $c_{1}$ existe. Parmi les deux branches $c_{1}$ et $c_{3}$, une seule appartient à la sphère; dans le cas de la figure 3 c'est la courbe $c_{3}$. Les calculs de [12] montrent que le positionnement des courbes dépend de la courbure de Gauss de la métrique Riemannienne restreinte au plan $(x, y)$, évaluée en 0 . Les deux situations sont identifiées en termes de vecteurs adjoints, c'est l'équivalent de la relation $p=n \theta$ en optique géométrique, voir Fig.4.

Les contacts des courbes se calculent dans la catégorie polynomiale. Les courbes $c_{i}$ sont tangentes à la direction anormale car le cône de Pontriaguine $K(r)$ coïncide dans la direction anormale avec le plan $(x, y)$. Les estimations sont les suivantes :

Proposition 4. Posons $Z=\frac{z}{r^{3}}$ et $X=\frac{x+r}{2 r}, r$ assez petit. Alors :

- $c_{1}: Z=\left(\frac{1}{6}+O(r)\right) X^{3}+o\left(X^{3}\right)$ 


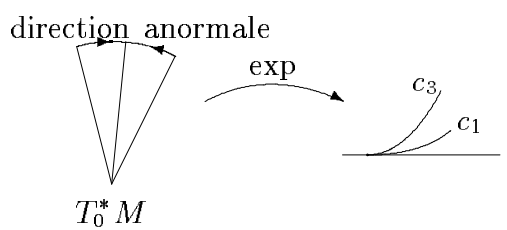

FIG.4.

- $c_{2}: Z=-\frac{2}{r^{2} \alpha^{2}} X^{2}+o\left(X^{2}\right)$

On en déduit :

Proposition 5. Dans le cas strict, la boule $B(0, r)$ de petit rayon a au voisinage de la direction anormale les propriétés suivantes :

1. elle est l'image par l'application exponentielle d'un secteur non compact du cylindre $(\lambda \rightarrow \infty)$.

2. elle est homéomorphe à un secteur conique centré sur $L$ et est en particulier simplement connexe.

3. elle est formée de feuilles $c_{2}, \bar{c}_{1}\left(=c_{1}\right.$ ou $\left.c_{3}\right)$ associées aux sphères $S(0, \varepsilon)$, $\varepsilon \leqslant r$ qui se recollent le long de la direction anormale, voir Fig. 5 .

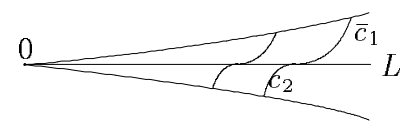

FIG.5.

La transcendance du secteur. C'est un secteur non sous-analytique où les calculs nécessitent l'usage des fonctions hyperboliques. Dans le cas conservatif les calculs utilisent la catégorie log-exp et la théorie de l'élimination dans cette catégorie, pour les détails voir [12], [28]. Le calcul de la sphère et donc du bord du domaine est délicat car il y a un phénomène de compensation qui s'explique à l'aide des invariants micro-locaux du secteur.

Invariants micro-locaux du passage du col. Dans le cas plat, les deux valeurs propres du pendule linéarisé au voisinage de $(-\pi, 0)$ sont $(-1,+1)$ et sont donc résonantes. En perturbant génériquement on obtient un spectre en bandes $\pm 1+\mathrm{O}\left(\frac{1}{\sqrt{\lambda}}\right)$ et les calculs dans le secteur utilisent tout le spectre, d'où en particulier un phénomène de stabilité. Par contre pour calculer la sphère il faut tenir compte de l'interaction entre toutes les valeurs propres du spectre pour en déduire une moyenne et c'est beaucoup plus complexe. 
Dans nos calculs on a privilégié la coupe de $B(0, r)$ avec le plan $y=0$ pour des raisons géométriques, mais on peut généraliser aisément.

Definition 1. On appelle 2-secteur de Martinet de la boule sous-Riemannienne l'intersection de la boule $B(0, r)$ avec un 2-plan qui contient la direction anormale.

Les calculs montrent que les propriétés de la proposition 4 se généralisent à tout secteur de Martinet :

Proposition 6. Tout secteur de Martinet est différentiablement représenté par la figure 6. Dans le cas conservatif des calculs explicites permettent d'évaluer son bord $S(0, r)$ qui est en particulier non sous-analytique.

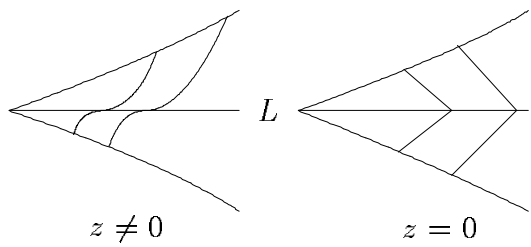

FIG.6.

Remarque. On conjecture évidemment que la non sous-analyticité de la sphère reste vraie en général mais les calculs dans le cas non conservatif sont complexes et non standards; en particulier il faut utiliser des cartes comme pour les calculs des cycles limites, et il y a un problème de matching.

Cut-locus. Les calculs du cas conservatif conduisent à conjecturer que la trace du lieu de coupure $L(0)$ sur la sphère, au voisinage de la direction anormale, est représentée sur la Fig.7.

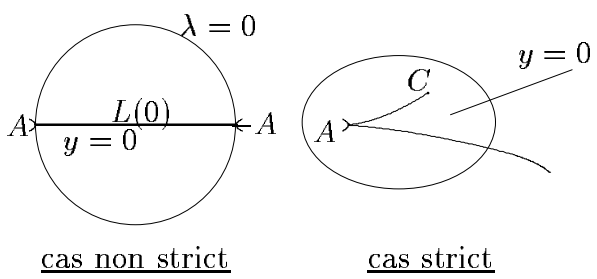

FIG.7.

Dans le cas non strict il y a deux branches se ramifiant en $A$. L'extrémité $C$ de la branche courte est un point conjugué. On peut le faire disparaître en restreignant la taille du voisinage et l'observateur voit alors deux branches 
de même taille. La taille de la grande branche est d'ordre $r$ et c'est donc très différent du cas de contact.

Eclatement en droites. Les résultats descriptifs présentés ici reposent sur des calculs longs et complexes dont la difficulté principale provient de la non validité du théorème de préparation lisse au voisinage de la direction anormale. C'est une difficulté d'ordre technique. Une bonne compréhension géométrique du problème peut reposer simplement sur l'idée d'éclater en droites, en plans, etc, l'origine, comme en section 5, et d'étudier la distance sousRiemannienne par rapport à ces objets, en utilisant la condition de transversalité. Cette idée guide d'ailleurs la représentation des variétés Lagrangiennes par des familles génératrices.

L'analyse micro-locale traduit le fait que dans la sphère sous-Riemannienne toutes les directions ne sont pas identiques et qu'il faut calculer par secteurs : secteur Riemannien, secteur de contact, secteur de Martinet... Dans chaque secteur les calculs sont différents. Par exemple dans le secteur de Martinet il faut utiliser les fonctions hyperboliques. On conçoit donc qu'en général une paramétrisation analytique de la sphère est impossible. On conjecture néanmoins l'existence d'une classe de Gevrey uniforme pour chaque problème sous-Riemannien et donc la possibilité de résoudre l'équation d'HamiltonJacobi-Bellman dans cette catégorie.

\section{Références}

1. Agrachev A. (1999) Compactness for sub-Riemannian length minimizers and subanalyticity, Report SISSA, Trieste.

2. Agrachev A., Sarychev A. V. (1995) Strong minimality of abnormal geodesics for 2-distributions, Journal of Dynamical and Control Systems, Vol. 1, No. 2, 139-176.

3. Agrachev A., Gamkrelidze R. V. (1997) Feedback invariant control theory and differential geometry I, Regular extremals, Journal of Dynamical and Control Systems, Vol. 3, No. 3, 343-390.

4. Agrachev A. and al. (1997) Sub-Riemannian spheres in the Martinet flat case, ESAIM/COCV, Vol. 2, 377-448.

5. Arnold V. (1976) Méthodes mathématiques de la mécanique classique, Eds Mir, Moscou.

6. Arnold V. and al., Singularities of differentiable mappings, Eds Mir, Moscou.

7. Bellaïche A. (1996) Tangent space in sub-Riemannian geometry, SubRiemannian Geometry, Birkhäuser.

8. Bliss G. A. (1946) Lectures on the calculus of variations, U. of Chicago Press, Chicago.

9. Bonnard B., Chyba M. (1999) Méthodes géométriques et analytiques pour étudier l'application exponentielle, la sphère et le front d'onde en géométrie sousRiemannienne de Martinet, ESAIM/COCV, Vol. 4, 245-334. 
10. Bonnard B., Kupka I. (1993) Théorie des singularités de l'application entrée/sortie et optimalité des trajectoires singulières dans le problème du temps minimal, Forum Math. 5, 111-159.

11. Bonnard B., de Morant J. (1995) Towards a geometric theory in the timeminimal control of chemical batch reactors, SIAM Journal on Control and Optimization, Vol. 33, No. 5, 1279-1311.

12. Bonnard B., Trélat E. (1999) Role of abnormal minimizers in sub-Riemannian geometry, PrePrint Dijon.

13. Gromov M. (1996) Carnot-Carathéodory spaces seen from within, SubRiemannian Geometry, Birkhäuser.

14. Guillemin V., Sternberg S. (1984) Symplectic techniques in physics, Cambridge University Press.

15. Hörmander L. (1983) The analysis of linear partial differential operators, Springer-Verlag, New-York.

16. Jean F. (1999) Entropy and complexity of a path in sub-Riemannian geometry, rapport ENSTA.

17. Kerkovian J., Cole J. D. (1981) Perturbation methods in applied mathematics, Springer-Verlag.

18. Kupka I. (1992) Abnormal extremals, Preprint.

19. Kupka I. (1996) Géométrie sous-Riemannienne, Séminaire Bourbaki, Paris.

20. Lawden D. F. (1989) Elliptic functions and applications, Springer-Verlag, NewYork.

21. Liu W. S., Sussmann H. J. (1995) Shortest paths for sub-Riemannian metrics of rank two distributions, Memoirs AMS, N564, Vol. 118.

22. Mischenko A. S. and al. (1990) Lagrangian manifolds and the Maslov operator, Springer-Verlag.

23. Moyer H. G. (1973) Sufficient conditions for a strong minimum in singular problems, SIAM Journal on Control and Optimization, 11, 620-636.

24. Naimark M. A. (1967) Linear differential operators, Frederick U. Pub. Co.

25. Nikiforov A., Ouranov V. (1982) Fontions spéciales de la physique mathématique, Eds Mir.

26. Ramis J. P., Séries divergentes et théorie asymptotique, Mémoires de la SMF.

27. Roussarie R. (1968) Bifurcations of planar vector fields and Hilbert's 16th problem, Birkhäuser, Berlin.

28. Trélat E. (2000) Some properties of the value function and its level sets for affine control systems with quadratic cost, to appear in Journal of Dynamical and Control Systems, Oct. 2000.

29. Treves F., Symplectic geometry and analytic hypo-ellipticity, Preprint. 